\title{
Sorafenib in the treatment of thyroid cancer
}

Silvia Martina Ferrari ${ }^{1}$, Ugo Politti ${ }^{1}$, Roberto Spisni ${ }^{2}$, Gabriele Materazzi ${ }^{2}$, Enke Baldini ${ }^{3}$, Salvatore Ulisse ${ }^{3}$, Paolo Miccoli ${ }^{2}$, Alessandro Antonelli ${ }^{1 *}$, Poupak Fallahi ${ }^{1}$.

${ }^{1}$ Department of Clinical and Experimental Medicine, University of Pisa, Via Savi, 10, 56126, Pisa, Italy;

${ }^{2}$ Department of Surgical, Medical, Molecular Pathology and Critical Area, University of Pisa, Via Savi, 10, 56126, Pisa, Italy;

${ }^{3}$ Department of Experimental Medicine, 'Sapienza', University of Rome, Viale dell’Università 30, 00185 Rome, Italy.

\section{*Corresponding Author:}

Prof. Alessandro Antonelli

Department of Clinical and Experimental Medicine

University of Pisa

Via Savi, 10, 56126, Pisa, Italy

E-mail: alessandro.antonelli@med.unipi.it

Tel.: +39-050-992318; Fax: +39-050-553235

Silvia Martina Ferrari

Ugo Politti

Roberto Spisni

Gabriele Materazzi

Enke Baldini

Salvatore Ulisse

Paolo Miccoli

Alessandro Antonelli

Poupak Fallahi sm.ferrari@int.med.unipi.it

upolitti@yahoo.it

roberto.spisni@med.unipi.it

g.materazzi@med.unipi.it

enke.baldini@uniroma1.it

salvatore.ulisse@uniroma1.it

paolo.miccoli@dc.unipi.it

alessandro.antonelli@med.unipi.it

poupak@int.med.unipi.it 


\section{Summary}

Sorafenib has been evaluated in several phase II and III studies in patients with locally advanced/metastatic radioactive iodine (RAI)-refractory DTC, reporting partial responses, stabilization of the disease, and improvement of progression free survival. Best responses were observed in lung metastases, and minimal in bone lesions. On the basis of these studies sorafenib was approved for the treatment of metastatic differentiated thyroid carcinoma in November 2013. Few studies suggested that reduction of Tg levels, or of average SUV at the FDG-PET, could be helpful for the identification of responding patients; but further studies are needed to confirm these results. Tumor genetic marker levels did not have any prognostic or predictive role in DTC patients. Commonest adverse events include skin toxicity, gastrointestinal and constitutional symptoms. Encouraging results have been also observed in patients with MTC. Many studies are ongoing to evaluate long term efficacy and tolerability of sorafenib in DTC patients.

Keywords: sorafenib, tyrosine kinase inhibitors, papillary thyroid cancer, follicular thyroid cancer, anaplastic thyroid cancer, medullary thyroid cancer. 


\section{Introduction}

Thyroid cancer (TC) represents the predominant endocrine malignancy $\left(7^{\text {th }}\right.$ most common cause of new cancer in the US for women (in 2007); $14^{\text {th }}$ in men). The incidence of papillary thyroid cancer (PTC) is increasing in the last decades [1-4].

The reasons of this increasing incidence are not clear. The wide use of neck ultrasonography and fine needle aspiration (FNA) of thyroid nodules is partially related to an increasing diagnosis of small TC. Among risk factors for TC, ionizing radiations play an important role, in particular considering exposure in the early life (about $2-4 \%$ of the subjects exposed to radiation during childhood or adolescence show TC) [5]. The exposure to nuclear explosions or nuclear accidents constitutes a risk factor for TC [6]. In fact, the incidence of TC rapidly increased in childhood, after the nuclear accident of Chenobyl (in 1986), in the regions of Belarus and Ukraine, exposed to the radiation fallout [7]. Even low doses of radiation exposure might lead to the onset of thyroid nodules and cancer [8]. Iodine deficiency has been suggested to be associated with a higher frequency of follicular thyroid cancer (FTC) [9]. Recently, also autoimmune thyroiditis has been shown to be a risk factor for PTCs $[10,11]$. Furthermore, new risk factors are emerging in the last decades [12].

While the incidence of TC is increasing in the last decades, the prevalence of TC deaths per year (in relation to the number of new cases) is decreased from $15 \%$ to $5 \%$ [13].

Differentiated thyroid carcinomas (DTC) that arise from follicular cells account for more than $90 \%$ of all thyroid tumors, and are subdivided into PTC or FTC according to histopathological criteria. Medullary thyroid cancer (MTC) accounts for $<5 \%$ of TC [1].

Completion total thyroidectomy is the treatment of choice for PTCs and FTCs [14].

Radioiodine is not routinely recommended in low risk DTC patients, and it should be considered in intermediate risk (IR), and it is routinely recommended in high-risk (HR) patients $[15,16]$.

After surgery (and eventually radioiodine) patients with PTC and FTC are followed by the determination of basal or thyroid-stimulating hormone (TSH)-stimulated thyroglobulin (Tg), and by 
neck ultrasonography [17-19].

More than $90 \%$ of patients with DTC has a normal life expectancy [20]. Only about 5\% of DTC patients have distant metastasis at the diagnosis (in the lungs, or bones, or other sites). However, during the follow-up about $10 \%-15 \%$ of patients have recurrent disease (in the thyroid bed, or lymph nodes), with a survival reduction (from $70 \%$ to $50 \%$, at 10 -year) [21, 22].

During tumor progression, DTC cancer cells may lose the iodide uptake ability, and TC changes into resistant to radioiodine, while prognosis significantly worsens [20-22].

Radiotherapy is of limited efficacy in the treatment of dedifferentiated thyroid cancer [23]. The only approved chemotherapy in most countries is doxorubicin, but partial or complete responses are rare and the toxicity profile is not favorable.

\subsection{Molecular pathways involved in DTC progression}

During the last decades, several somatic mutations in different molecular pathways in TC have been shown, and associated with its development and progression.

Activating RET (REarranged during Transfection) mutations and rearrangements have been identified in various human cancers [24]. RET is a proto-oncogene located on 10q11.2, encoding a transmembrane protein harboring a tyrosine kinase (TK) at the intracellular region. In approximately $40 \%$ of adult sporadic PTC, RET/PTC rearrangements are present [25]. The most frequent rearrangements are RET/PTC1, and RET/PTC3. RET/PTC rearrangements are frequently found in microcarcinomas and in benign thyroid lesions, so it has been hypothesized that RET/PTC rearrangements are determinant for the tumor initiation, but not for its progression [26-29].

The serine-threonine kinase BRAF is a member of the RAF family proteins. A substitution from valine to glutamate at residue 600 (V600E) of BRAF is present approximately in $45 \%$ of PTC, and it has been correlated with the tumor aggressiveness, and the loss of iodide uptake [25].

Recently mutations in the promoter of the telomerase reverse transcriptase (TERT) gene have been 
found in $11 \%$ PTCs, $11 \%$ of FTCs, $37 \%$ of poorly differentiated thyroid cancers (PDTC), and $42 \%$ of ATCs. These studies first showed that TERT promoter mutations in TCs are particularly prevalent in the aggressive TCs, overall in presence of BRAF mutations [30, 31].

K-RAS, N-RAS and H-RAS belong to the RAS (from "Rat Sarcoma") gene family, encoding intracellular G-proteins involved in activation of different intracellular signaling pathways. RAS mutations occur in $\sim 10 \%$ of PTCs, and in about $40-50 \%$ of FTCs. The association between RAS mutations and a more aggressive behaviour of TC has been shown [32].

About $30-40 \%$ of FTC show PAX8/PPAR $\gamma$ rearrangements, as $2-10 \%$ of follicular adenomas; these rearrangements are less common in the follicular variant of PTC [25].

TCs are more vascularized than normal thyroid tissue, and there is a unambigouous correlation between increased angiogenesis and a more aggressive TC behavior [33].

The expression of angiogenesis stimulators and inhibitors in TC is associated with clinical features of the disease [34].

Some of these are Vascular Endothelial Growth Factor (VEGF)/VEGF receptor (VEGFR), Epidermal Growth Factor (EGF)/EGFR, platelet-derived growth-factor (PDGF)/PDGFR, fibroblast growth factor (FGF)/FGFR, and hepatocyte growth factor (HGF)/c-Met.

VEGF is overexpressed and its main receptor VEGFR-2 is generally upregulated in DTC [35] and it is involved in neoplastic growth, progression and aggressiveness. Systemic administration of antiangiogenic drugs that target VEGF pathway has become a therapeutic option for TC patients [36].

It has been suggested that EGFR is highly expressed in aggressive PTC with lymph node metastasis, and plays a role in the progression of TC [37], but these results are in contrast with findings from other studies [38].

\subsection{Molecular pathways in MTC}


Most cases of MTC (originating from the cells producing calcitonin) are sporadic, while about $20 \%$ of MTC cases belong to a multiple endocrine neoplasia syndrome type 2 (MEN2). MTC is a slow growing cancer, even if the 10 -year overall survival rate is $40-50 \%$ in the presence of metastatic disease. Surgery is the only curative modality available [39], and no effective therapies exist for metastatic MTC, as it is not responsive to external radiotherapy, radionuclide therapy, or chemotherapy.

Activating point mutations in RET are associated with the hereditary cancer syndromes. There are three subtypes: MEN2A, MEN2B, and familial medullary thyroid carcinoma. There is a marked correlation between the position of the RET point mutation and the phenotype of MTC.

Almost all hereditary cases of MTC and $30-50 \%$ of the sporadic ones show activating RET mutations [40], so RET is considered an important therapeutic target for MTC [41].

Also other genetic events are determinant for the pathogenesis of MTC, as indicated by the recent findings of H-RAS mutations in 13-56\% of RET-negative sporadic MTC and the activation of the mammalian target of rapamycin (mTOR) intracellular signaling pathway in hereditary MTC [4244].

\section{Tyrosine Kinase Inhibitors (TKIs)}

Many strategies have been developed to block TKs.

TKIs are generally small organic compounds that compete with the ATP-binding site of the catalytic domain of the TKs [45], inhibiting TK autophosphorylation and activation, and preventing intracellular signaling pathways activation. TKIs can be specific to one or several TKs; so, a single TKI may target multiple TKs [46]. Most TKIs are multikinase inhibitors, being not specific for one particular type of TK. Various TKIs have been evaluated in TC, in vitro, and in pre-clinical, and clinical studies.

TKI blockade may be achieved also through monoclonal antibodies against growth factors 
receptors.

TKIs act on the above mentioned pathways involved in angiogenesis, growth, invasiveness, and local and distant spread [47].

TKIs are emerging as effective therapies of aggressive TC. Since TKIs act on pathways that are not selective for a specific type of TC, they have been evaluated in various tumors, including DTC, MTC and ATC [48].

The Response Evaluation Criteria in Solid Tumors (RECIST) estimate the individual patient response considering the clinical trials evaluating TKIs [49].

\section{Sorafenib}

Sorafenib $\left(\right.$ Nexavar $^{\circledR}$ ) is a small molecular (a bi-aryl urea) multi-targeted TKI, approved for the treatment of primary kidney cancer [advanced renal cell carcinoma (RCC)] and advanced primary liver cancer [hepatocellular carcinoma (HCC)].

It demonstrated inhibitor activity against VEGFR-2 and 3, c-Kit, PDGFR, RET/PTC, and Raf kinases (more avidly C-Raf than B-Raf), and it is able to target the Raf/Mek/Erk pathway (MAPK pathway). In addition, sorafenib has been also shown to induce apoptosis through down-regulation of Mcl-1 in many cancer types [50, 51].

Sorafenib is approved in USA and UE for the treatment of RCC [51], and HCC [51]. Sorafenib has been also approved for the treatment of metastatic DTC in November 2013 [52].

\subsection{Pharmacokinetic (PK) Profile}

\subsubsection{Absorption and distribution}

The mean relative bioavailability of sorafenib tablets after their administration is $38-49 \%$ in comparison with an oral solution. The absolute bioavailability is still not clear. Upon oral administration, sorafenib reaches peak plasma concentrations in about 3 hours. The absorption of sorafenib decreased of about $30 \%$ if it is administered with a high-fat meal with respect of the 
administration in the fasted state. Mean C-max and area under curve increased less than proportionally beyond doses of $400 \mathrm{mg}$ bid. Sorafenib binds human plasma proteins in vitro with a probability of $99.5 \%$.

Multiple dosing of sorafenib for 7 days resulted in a 2.5- to 7-fold accumulation with respect to single dose administration. Steady state plasma sorafenib concentrations are achieved within 7 days, with a peak to trough ratio of mean concentrations $<2[51]$.

\subsubsection{Biotransformation and elimination}

Sorafenib is metabolized mainly in the liver and goes through oxidative metabolism, mediated by cytochrome P450 3A4 (CYP3A4), and glucuronidation by UGT1A9. Its elimination half-life is about 25-48 hours. Bacterial glucuronidases cleave sorafenib conjugates in the gastrointestinal tract, permitting the reabsorption of the unconjugated drug. Considering an oral administration of $100 \mathrm{mg}$ of a solution formulation of sorafenib, $96 \%$ of the dose was recovered within 14 days, with $77 \%$ of it excreted in faeces, and $19 \%$ in urine as glucuronidated metabolites. The $51 \%$ of the dose accounts for unchanged sorafenib; it was excreted in faeces but not in urine, suggesting that biliary excretion of unchanged drug might contribute to the elimination of sorafenib.

Sorafenib accounts for approximately $70-85 \%$ of the circulating analytes in plasma at steady state. Eight metabolites of sorafenib are known, among which five have been revealed in plasma, and the main one is the pyridine N-oxide, showing in vitro potency similar to that of sorafenib. This metabolite represents about $9-16 \%$ of circulating analytes at steady state.

Neither age nor gender and race influence sorafenib PK.

Subjects with normal renal function, mild, moderate or severe renal impairment, do not show any relationship between sorafenib exposure and renal function.

The PK of sorafenib in Child-Pugh A and B non-HCC patients were similar to the PK in healthy volunteers, and no data are available for Child-Pugh C (severe) hepatic impairment. Sorafenib is primarily excreted through the liver, and exposure can be increased in this patient population [51]. 


\subsubsection{Drugs interactions}

Sorafenib is metabolized by CYP3A4 such as sunitinib, pazopanib and vandetanib and it seems to be the more susceptible to CYP3A4 inducers or inhibitors.

CYP2B6, CYP2C8 and CYP2C9 are inhibited in vitro by sorafenib with almost the same potency. In clinical PK studies, the concurrent administration of sorafenib $400 \mathrm{mg}$ bid with the CYP2B6 substrate cyclophosphamide, or paclitaxel, a CYP2C8 substrate, did not show a clinically meaningful inhibition. For this reason, sorafenib at the recommended dose of $400 \mathrm{mg}$ bid could not be an in vivo inhibitor of CYP2B6 or CYP2C8.

Moreover, the concurrent administration of sorafenib and the CYP2C9 substrate warfarin did not evidence changes in mean prothrombin time-international normalized ratio with respect to placebo. For this reason, the risk for a clinically relevant in vivo inhibition of CYP2C9 by sorafenib could be low. However, patients treated with warfarin or phenprocoumon should have their international normalized ratio checked regularly.

The concurrent treatment with sorafenib and midazolam, dextromethorphan or omeprazole (substrates for cytochromes CYP3A4, CYP2D6 and CYP2C19, respectively) did not alter their exposure, suggesting sorafenib is neither an inhibitor nor an inducer of these cytochrome P450 isoenzymes. For this reason, clinical PK interactions of sorafenib with these substrates are uncommon.

As already described above, although sorafenib is metabolized by CYP3A4, which can be inhibited or induced by various other drugs and environmental chemicals, more than half of the elimination of the drug is represented by biliary excretion of the unchanged parent drug, and this kind of elimination is not affected by agents inhibiting CYP3A4 activity. A paper evaluating the concurrent treatment with the CYP3A4 inhibitor ketoconazole and sorafenib in healthy male subjects did not evidence any change in the sorafenib PK.

The metabolism of sorafenib can be increased by strong CYP3A4 inducers, but this possible 
interaction has not been assessed by clinical studies [51].

Recently, it was demonstrated a synergistic anti-proliferative effect of metformin and sorafenib: the addition of metformin enabled a significative reduction of sorafenib dose without loss of its growth inhibitory efficacy. This treatment could be used to reduce dose-related toxic side-effects [53].

\subsubsection{Protein p-glycoprotein-substrates}

Sorafenib is able to inhibit the transport protein p-glycoprotein (P-gp) in vitro. Increased plasma concentrations of P-gp substrates such as digoxin cannot be excluded with the concurrent treatment with sorafenib. In vitro, the glucuronidation is inhibited by sorafenib through UGT1A1 and UGT1A9 [51].

The clinical significance of this inhibition is not completely known and drugs that are metabolized by these enzymes should be used with caution in patients treated with sorafenib owing to a potential risk of drug interactions.

Sorafenib has a moderate affinity for several members of the ATP-binding cassette sub-family $(\mathrm{ABC})$.

In LLC-PK1, Caco-2, K562, and MDCKII cells, it was shown that sorafenib is a moderate substrate for the efflux transporter ABCB1 (P-glycoprotein) and ABCG2 (breast cancer resistance protein) [54].

It has been shown in vivo that sorafenib is a substrate of ABCG2 and probably also of ABCB1. Mice completely lacking both functional above mentioned transporters and administered with oral sorafenib had a higher accumulation of this drug in the brain than mice lacking either of the transporters alone.

\subsubsection{Combination with other anti-neoplastic agents}

Sorafenib has been administered with other anti-neoplastic agents in clinical studies, including gemcitabine, cisplatin, oxaliplatin, paclitaxel, carboplatin, capecitabine, doxorubicin, irinotecan, 
docetaxel and cyclophosphamide, demonstrating no clinically relevant effect on the PK of gemcitabine, cisplatin, oxaliplatin or cyclophosphamide.

No evident drug interactions between sorafenib and carboplatin, dacarbazine, gemcitabine, oxaliplatin, and paclitaxel was observed in phase I clinical trials. However, the doxorubicin area under curve was $47 \%$ greater upon co-administration with sorafenib, even if no significant difference in toxicity was observed in spite of the greater exposure to doxorubicin. If coadministered with irinotecan, exposure was greater for both irinotecan $(26 \%-42 \%)$ and its active metabolite SN-38 (70\%-120\%), but the diarrhea resulting from irinotecan did not present an appreciable worsening with the combination [51].

\subsubsection{Sorafenib and other targeted agents}

A phase I dose-escalation trial of sorafenib and bevacizumab was performed on 39 patients with various cancers at below-recommended single-agent doses [55]. This combination showed promising clinical activity in particular in ovarian cancer, but the rapidity and frequency of dose reductions indicated that long-term dosage was not tolerated, and the need for alternative dosing schedules.

The intermittent sorafenib dosing with bevacizumab has been evaluated by a phase I study and evidenced a clinical activity; a few patients required a sorafenib dose reduction and showed few side effects [56].

The combination of sorafenib with erlotinib was investigated in a phase I trial: it was well tolerated and showed promising activity [57]. In a phase II trial for the combination, a higher progressionfree survival (PFS) and OS was seen in the EGFR wild type and the EGFR FISH-negative patients with advanced non-small cell lung cancer, compared to erlotinib alone [58]. However, the benefit of this combination needs to be confirmed by additional researches. A pre-clinical study on the combination of sorafenib with erlotinib or cetuximab has shown synergistic antitumor activity in both colorectal cancer and non-small cell lung cancer [59]. 
A phase I study evaluated sorafenib plus interferon (IFN)- $\alpha-2 \mathrm{a}$ in advanced RCC and melanoma and it evidenced preliminary antitumor activity and it was well-tolerated [60]. Another study was conducted on the combination of sorafenib and IFN- $\alpha-2 b$ in advanced RCC patients and it showed substantial activity, but the toxicity was higher than the one of either drug alone [61]. However, dose reductions and breaks between cycles allowed for long term therapy. On the contrary, more recently, a phase II study, evaluating the combination of sorafenib and pegylated IFN- $\alpha-2 b$ in metastatic melanoma patients, evidenced a modest clinical activity and severe side effects, such as fatal bleeding complications [62].

However, the combination of sorafenib with other different targeted agents could be associated with important adverse events (AEs). In fact, it has been reported a case of fatal heart failure after a 26months combination of two TKIs (sorafenib and imatinib) in a patient treated due to chronic myeloid leukemia and PTC [63].

\subsection{Sorafenib in TC in vitro studies}

Carlomagno et al. [64] first showed that sorafenib is capable of inhibiting oncogenic RET mutants, demonstrating that it prevented the growth of the TPC1 and TT, thyroid carcinoma cell lines, with the RET/PTC1 and C634W RET mutation, respectively.

These results were subsequently confirmed in the PTC cells carrying the RET/PTC1 rearrangement, that resulted more sensitive to sorafenib with respect to PTC cells carrying BRAF mutation [65]. More recently it has been shown that sorafenib had important effects on cell proliferation, cell cycle arrest, and signal transduction pathways in PTC cells harboring RET/PTC1 rearrangement, and that it could be potentially used to enhance the expression of iodide-handling genes and to inhibit the expression of glucose transporter genes [50].

Sorafenib exerted antineoplastic activity in the other human MTC cell line (MZ-CRC-1), too [66]. Moreover, it has been also demonstrated that sorafenib has antineoplastic action in vitro, and 
inhibits the angiogenesis and growth of orthotopic xenografts of ATC in nude mice [67].

This drug, therefore, potentially may inhibit TC growth via anti-proliferative and anti-angiogenic mechanisms.

\section{Sorafenib clinical studies}

\subsection{Phase II studies (Table I)}

A phase II trial, published in 2008, assessed the efficacy of sorafenib in 30 patients with metastatic, iodine-non-avid, TC, including DTC, PDTC, MTC, and ATC subtypes. The drug was administered at $400 \mathrm{mg}$ bid; the median duration of treatment was 27 weeks. Six patients (20\%) discontinued treatment owing to AEs. Doses were reduced in $47 \%$ of patients (14 patients) to control toxicities. A partial response (PR) rate was observed in $23.3 \%$ and a stable disease (SD) rate in $53.3 \%$, with a clinical benefit rate (partial response + stable disease) of $77 \%$, and a median progression-free survival (PFS) of 21 months [68].

Few months later it was published a study in 58 patients with metastatic TC ( 25 chemotherapy naive patients with metastatic PTC; 33 patients with metastatic PTC previously treated with chemotherapy, or other subtype as Hurthle cell, Follicular, ATC, or mixed TC). Patients were treated with sorafenib at $800 \mathrm{mg}$ daily (in two doses; generally well tolerated). It was demonstrated that sorafenib has clinical antitumor activity in metastatic TC, as a PR was observed in 6 patients, while 23 patients had SD longer than 6 months [69].

A prospective phase II study assessed the efficacy of sorafenib in reinducing radioactive iodine (RAI) uptake in patients with progressive metastatic or locally advanced RAI refractory DTC. Thirtyone patients received sorafenib $400 \mathrm{mg}$ twice daily, for 36 weeks: $25 \%$ had a PR and 34\% a SD, the estimated PFS was 58 weeks. However, 22\% of patients had progressive disease and diagnostic whole body scan did not evidence any reinduction of RAI uptake. The efficacy of sorafenib was lower in patients with bone metastases [70].

Brose et al. published another open-label phase II study of sorafenib at maximum dose ( $800 \mathrm{mg}$ 
daily) in metastatic iodine-refractory TC, in 55 patients with different histological subtypes (47\% PTC, 36\% FTC/Hürthle Cell, 8\% MTC, 9\% PDTC/ATC). Genotyping of BRAF was complete in 16 patients. PFS increased in PTC patients with B-RafV600E, with respect to wild-type B-Raf (84 versus 54 weeks, $\mathrm{P}=0.028$ ) [71]. A paper published in 2010 reports the M. D. Anderson Cancer Center's experience with the off-label use of TKs inhibitors sorafenib and sunitinib for refractory to iodine DTC (PTC and FTC). Sorafenib was used in 13 patients, at the same dose of the previous reported trials. The obtained data were similar to those of other phase II studies evaluating sorafenib in TC (remission rate of $20 \%$, durable response rate of $66 \%$, and a clinical benefit rate of $80 \%$; PFS was 19 months, and the median overall survival at 2 years was $67 \%$ ). A response variability to sorafenib of the different metastases in the same patient was observed, with the best response in lung, and minimal in non-irradiated bone lesions, suggesting a differential expressions and inhibitions of various receptors. After the therapy, Tg levels were reduced, and a correlation between the $\log \mathrm{Tg}$ and the response to the treatment was demonstrated (the reduction preceded tumor shrinkage), suggesting that $\mathrm{Tg}$ would be a good biologic marker of response to therapy [72]. Another phase II study was conducted in 19 patients with metastatic MTC and 15 patients with locally advanced radioiodine refractory DTC. The radiological response rate (RR) was $18 \%$ for patients with DTC, while the PFS at 2 years was $62 \%$ and OS $72 \%$. However, $79 \%$ of patients required dose reduction for $\mathrm{AE}$ (hand-foot syndrome, other skin toxicities, diarrhoea and alopecia). A patient who had demonstrated a dramatic response after 3 months of therapy showed a mutation in BRAF exon 15 [73].

In the 2011 ASCO annual meeting, the results of UPCC 03305 phase II trial of sorafenib for advanced thyroid carcinoma were presented. Fifty-five patients $(85 \%$ DTC/PD, 9\% ATC, 6\% MTC) received the drug at same dose provided in preceding studies. A longer PFS was observed in patients with DTC/PD (96 weeks versus 93.6 weeks) (38\% achieved a PR, 47\% had SD) [74].

Capdevila et al. conducted a retrospective analysis of patients with metastatic TC treated with sorafenib in seven Spanish referral centers. Thirthy-four patients were included between June 2006 
and January 2010. They were all not suitable for any traditional therapies and received $400 \mathrm{mg}$ twice a day sorafenib. Sixteen patients presented DTC (7 papillary, 9 follicular), 15 MTC, and 3 ATC. Eleven patients (32\%) achieved PR and 14 (41\%) had SD beyond 6 months. RRs were 47\% (7 of 15) for MTC, 19\% (3 of 16) for DTC and 33\% (1 of 3) for ATC. With a median follow-up of 11.5 months, mPFS were 13.5, 10.5 and 4.4 months for DTC, MTC and ATC respectively. A significant decrease in tumor markers ( $\mathrm{Tg}$, calcitonin and carcinoembryogenic antigen) was also observed [75].

Another phase II trial of sorafenib (400 mg bid) was conducted in 31 patients with advanced radioiodine refractory DTC; the median follow-up and period of treatment was 25 and 9 months, respectively. Among the mutations found, BRAF V600E was the most present, but it was not related to disease progression. PR was seen in $31 \%$ of patients and $45 \%$ achieved SD after a median follow-up of 25 months. The dose of TKI used was generally well tolerated, although dose reductions were required in $58 \%$ of patients [76].

Savvides et al. treated 20 ATC patients with sorafenib $400 \mathrm{mg}$ bid. Two of them had a PR (10\%) and an additional 5 of $20(25 \%)$ had SD. The duration of response (in the two responders) was 10 and 27 months, respectively. The median duration in SD was 4 months (range 3-11 months). The overall mPFS was 1.9 months (with a median and 1-year survival, of 3.9 months, and 20\%, respectively). Toxicity was manageable as previously described, including hypertension and skin rash [77].

Recently, the results obtained in a retrospective, longitudinal study on use of sorafenib in patients with progressive RAI-refractory DTC were published (sorafenib has been used off-label in 17 patients, at conventional dose). Thirty $\%$ of patients achieved PR, $41 \%$ SD and $18 \%$ PD, median PFS was 9 months and median OS 10 months. The drug was tolerated (however 5 fatal events were reported: 3 severe bleeding, 2 cardiac arrest), but all patients needed dose reductions and/or transient drug interruption to control AEs. These severe AEs have been attributed to the bad general conditions of the patients at the beginning of the trial (worse than in previous studies). The 
radiological response was greater in lymph nodes, than in lung metastasis. An early reduction in average SUV (Standardized Uptake Value) was recorded in all patients, but it was greater in responding subjects, suggesting that the FDG-PET (Fluorodeoxyglucose - Positron Emission Tomography) could be helpful for the timely identification of non-responding patients [78].

The role of sorafenib in the treatment of progressive metastatic DTC was subsequently confirmed by other studies, too [79].

Benekli et al. analyzed the results of the treatment in 14 DTC (PTC number $[\mathrm{n}]=10$; FTC $\mathrm{n}=4$ ) and 16 MTC. The median age was 57 years (range: 28-79 years), and there were 18 males and 12 females. All DTC patients were iodine-refractory and had been previously treated with radioactive iodine (range: 1-7 doses). Sorafenib was used for a median of 12 months (range: 1-49 months). The overall response rate was $20 \%$, all PRs (no complete response). The overall response rate was $14 \%$ in DTC and $25 \%$ in MTC patients. The median PFS was 17.1 months $(95 \%$ confidence interval [CI]: 7.3-26.8). The 2-year PFS and OS were 39\% and 68\%, respectively. DTC and MTC patients had similar survival outcomes: median PFS of 21.3 months (95\% CI: 5.8-36.7) versus 14.5 months (95\% CI: 3.7-25.2), respectively $(\mathrm{P}=0.36)$ (with the median OS not reached in either group). Tumor marker levels did not have any prognostic or predictive role. The sorafenib toxicity profile was similar to that of other trials [80].

Twenty patients with advanced RAI-refractory thyroid carcinoma were enrolled (March 2011March 2014) by Gallo et al. (sorafenib $400 \mathrm{mg}$ bid, tapering the dose in case of side effects). CT scans were performed every 3-4 months. Five patients stopped sorafenib within 90 days due to severe toxicities. Median PFS was 248 days. Five patients had a PR (achieved in all cases within 3 months), whereas 5 had SD at 12 months. Durable response rate (PR plus SD) for at least 6 months was $50 \%$, among those who received sorafenib for at least 3 months. Commonest AEs included skin toxicity, gastrointestinal and constitutional symptoms [81].

\subsection{Phase III studies}


On the basis of the phase II trials results, it has been conducted a multicenter, double-blind randomized phase III study, that evaluated the efficacy and safety of sorafenib with respect to placebo, in locally advanced/metastatic RAI-refractory DTC.

The DECISION trial was designed to evaluate the capability of sorafenib to improve PFS in patients with locally advanced or metastatic, RAI-refractory DTC (defined as a target lesion with no iodine uptake on a post RAI scan performed under conditions of a low iodine diet and adequate TSH elevation or recombinant human TSH stimulation). Efficacy and safety of sorafenib have been assessed every 56 days (two cycles) and 28 days ( 1 cycle) for the first 8 months and every 56 days after, respectively.

Patients have been randomized 1:1 to receive placebo or sorafenib. The inclusion criteria were: age $>18$, life expectancy of at least 12 weeks, locally advanced or metastatic DTC (PTC, FTC, Hurtle cell or PDTC) with at least one measurable lesion (measured by computer tomography or magnetic resonance imaging) and disease progression within 14 months. Some patients who have had some iodine uptake have been also included in the study.

Other inclusion criteria were: a performance status $<2$ according to Eastern Cooperative Oncology Group, adequate TSH suppression $(<0.5 \mathrm{mU} / \mathrm{L})$, absence of renal ad liver failure and adequate bone marrow function. Patients who had yet received any treatment with TKI, monoclonal antibodies against VEGFRs or other targeted agents, cytotoxic chemotherapy or thalidomide, were excluded.

The initial group comprises a population of 417 patients (207 treated with sorafenib and 210 with placebo), while the final group was constituted by 416 patients (207 treated with sorafenib and 209 with placebo). Median PFS was significantly higher in patients belonging to the sorafenib group (10.8 months) than in the placebo group (5.8 months). PFS improved in all pre-specified clinical and genetic biomarker subgroups, independently from mutation status [82].

Another phase III trial, conducted by Bayer, involving 417 patients, is still during [83].

\section{Safety and tolerability}


The most common AEs were: rash, alopecia and hand-foot syndrome, diarrhea; while the most important serious adverse reactions were: hypertension/hypertensive crisis, haemorrhage, myocardial infarction/ischaemia, gastrointestinal perforation, hepatitis.

Fatal events were also reported (bleeding, cardiac arrest) in few patients (attributed to the bad general conditions of the patients).

Undesirable effects are presented in the Table II.

\section{Limits and drug resistance}

The efficacy of sorafenib in DTC patients, even if promising, has given contrasting evidences in different clinical trials, probably owing to the drug resistance, that could arise from the activation of alternate mitogenic signals [84].

Sorafenib stops tumor growth, but does not remove tumor cells. Hence, the combination with other drugs, or with different TKIs has been recently proposed.

The co-administration of sorafenib with different anti-neoplastic agents at their commonly used dosing regimens, showed any clinically relevant effect on the PK of gemcitabine, cisplatin, oxaliplatin or cyclophosphamide.

In phase I clinical trials, dacarbazine, gemcitabine, oxaliplatin, doxorubicin and paclitaxel were administered with sorafenib without detectable drug interactions [85].

The combination of bevacizumab and sorafenib has been also reported (see 3.1.6) $[55,56]$, such as the combination of sorafenib with erlotinib (see 3.1.6) [57-59].

More recently, the combination of sorafenib with belinostat or panobinostat was studied in vitro in TC cells in vitro, showing no significant synergistic effect [86].

Attempts have been made to personalize the sorafenib therapy in each patient with DTC, on the basis of the molecular characterization of the tumor and of the host factors. Tumor genetic marker levels have not shown any prognostic or predictive role about sorafenib therapy in DTC patients.

The possibility to test the sensitivity of primary TC cells from each subject to sorafenib, or other 
different TKIs, might ameliorate the effectiveness of the treatment $[87,88]$.

Disease orientated in vitro drug screening, using primary human cancer cells, has predictive value for the clinical response activity [89, 90], and could prevent the administration of inactive drugs (potentially dangerous) to patients [91]. In fact, a positive (in vitro) chemosensitivity test permits to predict in vivo effectiveness in $60 \%$ of cases [92], while a negative chemosensitivity test (in vitro) is associated with a $90 \%$ of ineffectiveness of the chemotherapy in vivo [89].

Till now, primary TC cell cultures have been established only from surgical biopsies performed for therapeutic or diagnostic procedures. More recently, it has been demonstrated the possibility to obtain primary TC cell culture from FNA samples of ATC (FNA-ANA), overcoming surgical procedures, and opening the way to the use of FNA-ANA cells to test the sensitivity to different chemotherapeutics in each patient [56, 92-94].

\section{Conclusion}

Sorafenib has been evaluated in several phase II and III studies in patients with locally advanced/metastatic RAI-refractory DTC, reporting PRs, stabilization of the disease, and improvement of PFS. Until now it has not yet been demonstrated an improvement of overall survival. On the basis of these studies sorafenib in November 2013 was approved for the treatment of metastatic DTC [52]. A response variability of the different metastases in the same patient to sorafenib was observed in several studies, with best response in lung, and minimal in bone lesions. Few studies suggested that reduction of Tg levels, or of average SUV at the FDG-PET, could be helpful for the timely identification of responding patients; but further studies are needed to confirm these results. Tumor genetic marker levels did not have any prognostic or predictive role in DTC patients. Commonest AEs include skin toxicity, gastrointestinal and constitutional symptoms. Andecdotal studies continue to report positive results of sorafenib in DTC [95].

Encouraging results have been also observed in patients with MTC, reporting PRs, stabilization of 
the disease, improvement of PFS, and reduction of circulating markers such as calcitonin and CEA. However, further studies are needed to evaluate sorafenib in MTC.

Many studies are ongoing to evaluate long-term efficacy and tolerability of sorafenib in TC patients. However, further studies will be necessary to personalize the sorafenib therapy in each TC patient, on the basis of the molecular characterization of the tumor and of the host factors, to improve survival and the quality of life. 


\section{Expert commentary}

Sorafenib is active in patients with locally advanced/metastatic radioactive iodine (RAI)-refractory DTC, reporting PRs, stabilization of the disease, and improvement of PFS, and it is approved for the treatment of metastatic DTC. A response variability of the different metastases in the same patient to sorafenib was observed in different studies, with best response in lung, and minimal in bone lesions. Few studies suggested that reduction of Tg levels, or of average SUV at the FDG-PET, could be helpful for the timely identification of responding patients; but further studies are needed to confirm these results. Tumor genetic marker levels did not have any prognostic or predictive role in DTC patients. Commonest adverse events include skin toxicity, gastrointestinal and constitutional symptoms. Many studies are ongoing to evaluate long term efficacy and tolerability of sorafenib in DTC patients.

The treatment with sorafenib in DTC patients might lead to drug resistance, that could arise from the activation of alternate mitogenic signals. Hence, the combination with other drugs, or with different TKIs has been recently proposed.

Attempts have been made to personalize the sorafenib therapy in each patient with DTC, on the basis of the molecular characterization of the tumor and of the host factors, to improve survival and quality of life.

Promising results have been also observed in patients with MTC, reporting PRs, stabilization of the disease, improvement of PFS. However, further studies are needed to evaluate sorafenib in MTC.

\section{Five years view}

Many studies are ongoing to evaluate long-term efficacy and tolerability of sorafenib in DTC patients. These long term studies might finally clarify if sorafenib is able to prolonge OS in DTC patients.

Future studies will evaluate results about sorafenib in patients with MTC.

Other studies are evaluating the possibility to personalize the sorafenib therapy in each patient with 
DTC, on the basis of the molecular characterization of the tumor and of the host factors, to improve survival and quality of life.

\section{Key issues:}

1. Sorafenib (Nexavar ${ }^{\circledR}$ ) is a small molecular (a bi-aryl urea) multi-targeted TKI, demonstrating inhibitor activity against VEGFR-2 and 3, c-Kit, PDGFR, RET/PTC, and Raf kinases (more avidly C-Raf than B-Raf), and it is able to target the Raf/Mek/Erk pathway (MAPK pathway). In addition, sorafenib has been also shown to induce apoptosis through down-regulation of Mcl-1 in many cancer types.

2. Sorafenib was initially approved for the treatment of primary kidney cancer and advanced primary liver cancer.

3. Sorafenib has been evaluated in several phase II and III studies in patients with locally advanced/metastatic radioactive iodine (RAI)-refractory DTC, reporting partial responses, stabilization of the disease, and improvement of progression free survival.

4. Best responses were observed in lymph node and lung metastases, and minimal in bone lesions.

5. Commonest adverse events include skin toxicity, gastrointestinal and constitutional symptoms.

6. Sorafenib was approved for the treatment of metastatic differentiated thyroid carcinoma in November 2013.

7. Few studies suggested that reduction of Tg levels, or of average SUV at the FDG-PET, could be helpful for the identification of responding patients; but further studies are needed to confirm these results.

8. Attempts have been made to personalize the sorafenib therapy in each patient with DTC, on the basis of the molecular characterization of the tumor and of the host factors.

9. Promising results have been also observed in patients with MTC, reporting PRs, 
stabilization of the disease, improvement of PFS.

10. The treatment with sorafenib in TC patients might lead to drug resistance, that could arise from the activation of alternate mitogenic signals. Hence, the combination with other drugs, or with different TKIs has been recently proposed. 


\section{REFERENCES}

1. Cancer statistics highlight progress, challenges. Cancer Discov 2014;4:OF3

2. Colonna M, Uhry Z, Guizard A, et al Recent trends in incidence, geographical distribution, and survival of papillary thyroid cancer in France. Cancer Epidemiol 2015

3. Finlayson A, Barnes I, Sayeed S, et al. Incidence of thyroid cancer in England by ethnic group, 2001-2007. Br J Cancer 2014;110:1322-7

4. Enewold LR, Zhou J, Devesa SS, et al. Thyroid cancer incidence among active duty U.S. military personnel, 1990-2004. Cancer Epidemiol Biomarkers Prev 2011;20:2369-76

5. Schlumberger M, Chevillard S, Ory K, et al. Thyroid cancer following exposure to ionizing radiation. Cancer Radiother 2011;15:394-9

6. Braverman ER, Blum K, Loeffke B, et al. Managing terrorism or accidental nuclear errors, preparing for iodine-131 emergencies: a comprehensive review. Int J Environ Res Public Health 2014;11:4158-200

7. Antonelli A, Miccoli P, Derzhitski VE, et al. Epidemiologic and clinical evaluation of thyroid cancer in children from the Gomel region (Belarus). World J Surg 1996;20:867-71

8. Suzuki K, Yamashita S. Low-dose radiation exposure and carcinogenesis. Jpn J Clin Oncol $2012 ; 42: 563-8$

9. Zimmermann MB, Boelaert K. Iodine deficiency and thyroid disorders. Lancet Diabetes Endocrinol 2015;3:286-95

10. Jankovic B, Le KT, Hershman JM. Clinical Review: Hashimoto's thyroiditis and papillary thyroid carcinoma: is there a correlation? J Clin Endocrinol Metab 2013;98:474-82

11. Antonelli A, Ferri C, Fallahi P, et al. Clinical and subclinical autoimmune thyroid disorders in systemic sclerosis. Eur J Endocrinol 2007;156:431-37

12. Antonelli A, Ferri C, Fallahi P, et al. Thyroid cancer in HCV-Related chronic hepatitis patients: A case-control study. Thyroid 2007;17:447-51

13. Ward EM, Thun MJ, Hannan LM, Jemal A. Interpreting cancer trends. Ann N Y Acad Sci 
$2006 ; 1076: 29-53$

14. Miccoli P, Antonelli A, Spinelli C, et al. Completion total thyroidectomy in children with thyroid cancer secondary to the Chernobyl accident. Arch Surg 1998;133:89-93

15. Haugen BR. Radioiodine remnant ablation: current indications and dosing regimens. Endocr Pract 2012;18:604-10

16. Lamartina L, Cooper DS. Radioiodine remnant ablation in low-risk differentiated thyroid cancer: the "con" point of view. Endocrine 2015 Jan 10. [Epub ahead of print]

17. Antonelli A, Miccoli P, Ferdeghini M, et al. Role of neck ultra-sonography in the follow-up of patients operated on for thyroid cancer. Thyroid 1995;5:25-8

18. Antonelli A, Miccoli P, Fallahi P, et al. Role of neck ultra-sonography in the follow-up of children operated on for thyroid papillary cancer. Thyroid 2003;13:479-84

19. American Thyroid Association (ATA) Guidelines Taskforce on Thyroid Nodules and Differentiated Thyroid Cancer, Cooper DS, Doherty GM, et al. Revised American Thyroid Association management guidelines for patients with thyroid nodules and differentiated thyroid cancer. Thyroid 2009;19:1167-214

20. Verburg FA, Mäder U, Tanase K, et al. Life expectancy is reduced in differentiated thyroid cancer patients $\geq 45$ years old with extensive local tumor invasion, lateral lymph node, or distant metastases at diagnosis and normal in all other DTC patients. J Clin Endocrinol Metab 2013; 98:172-80

21. Gruber JJ, Colevas AD. Differentiated thyroid cancer: focus on emerging treatments for radioactive iodine-refractory patients. Oncologist 2015;20:113-26

22. Antonelli A, Fallahi P, Ferrari SM, et al. Dedifferentiated thyroid cancer: A therapeutic challenge. Biomed Pharmacother 2008;62:559-63

23. Sun XS, Sun SR, Guevara N, et al. Indications of external beam radiation therapy in nonanaplastic thyroid cancer and impact of innovative radiation techniques. Crit Rev Oncol Hematol 2013;86:52-68 
24. de Groot JW, Links TP, Plukker JT, et al. RET as a diagnostic and therapeutic target in sporadic and hereditary endocrine tumors. Endocr Rev 2006;5:535-60

25. Nikiforov YE. Thyroid carcinoma: molecular pathways and therapeutic targets. Mod Pathol 2008;21:S37-S43

26. Marotta V, Guerra A, Sapio MR, Vitale M. RET/PTC rearrangement in benign and malignant thyroid diseases: a clinical standpoint. Eur J Endocrinol 2011;165:499-507

27. Fagin JA. Challenging dogma in thyroid cancer molecular genetics- role of RET/PTC and BRAF in tumor initiation. J Clin Endocrinol Metab 2004;89:4264-6

28. Santoro M, Melillo RM, Fusco A. RET/PTC activation in papillary thyroid carcinoma: European Journal of Endocrinology Prize Lecture. Eur J Endocrinol 2006;155:645-53

29. Xing M. BRAF mutation in thyroid cancer. Endocrine-Related Cancer 2005;12:245-62

30. Liu X, Bishop J, Shan Y, et al. Highly prevalent TERT promoter mutations in aggressive thyroid cancers. Endocr Relat Cancer 2013;20:603-10

31. Xing M, Liu R, Liu X, et al. BRAF V600E and TERT promoter mutations cooperatively identify the most aggressive papillary thyroid cancer with highest recurrence. J Clin Oncol $2014 ; 32: 2718-26$

32. Howell GM, Hodak SP, Yip L. RAS mutations in thyroid cancer. Oncologist 2013;18:92632

33. Marotta V, Franzese MD, Del Prete M, et al. Targeted therapy with kinase inhibitors in aggressive endocrine tumors. Expert Opin Pharmacother 2013;14:1187-203

34. Tan A, Xia N, Gao F, et al. Angiogenesis-inhibitors for metastatic thyroid cancer. Cochrane Database Syst Rev 2010;3:CD007958

35. Bunone G, Vigneri P, Mariani L, et al. Expression of angiogenesis stimulators and inhibitors in human thyroid tumors and correlation with clinical pathological features. Am J Pathol $1999 ; 155: 1967-76$

36. Cao Y. VEGF-targeted cancer therapeutics-paradoxical effects in endocrine organs. Nat Rev 
Endocrinol 2014;10:530-9

37. Tang C, Yang L, Wang N, et al. High expression of GPER1, EGFR and CXCR1 is associated with lymph node metastasis in papillary thyroid carcinoma. Int J Clin Exp Pathol $2014 ; 7: 3213-23$

38. Lim DJ, Baek KH, Lee YS, et al. Clinical, histopathological, and molecular characteristics of papillary thyroid microcarcinoma. Thyroid 2007;17:883-8

39. Maxwell JE, Sherman SK, O'Dorisio TM, Howe JR. Medical management of metastatic medullary thyroid cancer. Cancer 2014;120:3287-301

40. Krampitz GW, Norton JA. RET gene mutations (genotype and phenotype) of multiple endocrine neoplasia type 2 and familial medullary thyroid carcinoma. Cancer 2014;120:1920-31

41. Antonelli A, Fallahi P, Ferrari SM, et al. RET TKI: potential role in thyroid cancers. Curr Oncol Rep 2012;14:97-104

42. Almeida MQ, Hoff AO. Recent advances in the molecular pathogenesis and targeted therapies of medullary thyroid carcinoma. Curr Opin Oncol 2012;24:229-34

43. Lyra J, Vinagre J, Batista R, et al. mTOR activation in medullary thyroid carcinoma with RAS mutation. Eur J Endocrinol 2014;171:633-40

44. Tamburrino A, Molinolo AA, Salerno P, et al. Activation of the mTOR pathway in primary medullary thyroid carcinoma and lymph node metastases. Clin Cancer Res 2012;18:3532-40

45. Lorusso PM, Eder JP. Therapeutic potential of novel selective-spectrum kinase inhibitors in oncology. Expert Opin Investig Drugs 2008;17:1013-28

46. Antonelli A, Fallahi P, Ferrari SM, et al. New targeted therapies for thyroid cancer. Curr Genomics 2011;12:626-31

47. Ye L, Santarpia L, Gagel RF. The evolving field of tyrosine kinase inhibitors in the treatment of endocrine tumors. Endocr Rev 2010;31:578-99

48. Jasim S, Ozsari L, Habra MA. Multikinase inhibitors use in differentiated thyroid 
carcinoma. Biologics $2014 ; 8: 281-91$

49. Therasse P, Arbuck SG, Eisenhauer EA, et al. New guidelines to evaluate the response to treatment in solid tumors. European Organization for Research and Treatment of Cancer, National Cancer Institute of the United States, National Cancer Institute of Canada. J Natl Cancer Inst 2000;92:205-16

50. Ruan M, Liu M, Dong Q, Chen L. Iodide- and glucose-handling gene expression regulated by sorafenib or cabozantinib in papillary thyroid cancer. J Clin Endocrinol Metab $2015 ; 100: 1771-9$

51. * Fallahi P, Ferrari SM, Santini F, et al. Sorafenib and thyroid cancer. BioDrugs 2013;27:615-28. An important review on pharmacology of sorafenib

52. ** Tuttle RM, Haddad RI, Ball DW, et al. Thyroid carcinoma, version 2.2014. J Natl Compr Canc Netw 2014;12:1671-80. NCCN Guidelines for thyroid carcinoma and its treatment

53. Chen G, Nicula D, Renko K, Derwahl M. Synergistic anti-proliferative effect of metformin and sorafenib on growth of anaplastic thyroid cancer cells and their stem cells. Oncol Rep $2015 ; 33: 1994-2000$

54. Lagas JS, van Waterschoot RA, Sparidans RW, et al. Breast cancer resistance protein and Pglycoprotein limit sorafenib brain accumulation. Mol Cancer Ther 2010;9:319-26

55. Azad NS, Posadas EM, Kwitkowski VE, et al. Combination targeted therapy with sorafenib and bevacizumab results in enhanced toxicity and antitumor activity. J Clin Oncol 2008;26:3709-14

56. Lee JM, Sarosy GA, Annunziata CM, et al. Combination therapy: intermittent sorafenib with bevacizumab yields activity and decreased toxicity. Br J Cancer 2010;102:495-9

57. Duran I, Hotté SJ, Hirte H, et al. Phase I targeted combination trial of sorafenib and erlotinib in patients with advanced solid tumors. Clin Cancer Res 2007;13:4849-57 
58. Spigel DR, Burris HA III, Greco FA, et al. Randomized, double-blind, placebo-controlled, phase II trial of sorafenib and erlotinib or erlotinib alone in previously treated advanced non-small cell lung cancer. J Clin Oncol 2011;29:2582-9

59. Martinelli E, Troiani T, Morgillo F, et al. Synergistic antitumor activity of sorafenib in combination with epidermal growth factor receptor inhibitors in colorectal and lung cancer cells. Clin Cancer Res 2010;16:4990-5001

60. Escudier B, Lassau N, Angevin E, et al. Phase I trial of sorafenib in combination with IFN $\alpha-2 a$ in patients with unresectable and/or metastatic renal cell carcinoma or malignant melanoma. Clin Cancer Res 2007;13:1801-9

61. Gollob JA, Rathmell WK, Richmond TM, et al. Phase II trial of sorafenib plus interferon $\alpha 2 \mathrm{~b}$ as first-or second-line therapy in patients with metastatic renal cell cancer. J Clin Oncol $2007 ; 25: 3288-95$

62. Egberts F, Gutzmer R, Ugurel S, et al. Sorafenib and pegylated interferon- $\alpha 2 b$ in advanced metastatic melanoma: a multicenter phase II DeCOG trial. Ann Oncol 2011;22:1667-74

63. Toubert ME, Vercellino L, Faugeron I, et al. Fatal heart failure after a 26-month combination of tyrosine kinase inhibitors in a papillary thyroid cancer. Thyroid $2011 ; 21: 451-4$

64. Carlomagno F, Anaganti S, Guida T, et al. BAY 43-9006 inhibition of oncogenic RET mutants. J Natl Cancer Inst 2006;98:326-34

65. Henderson YC, Ahn SH, Kang Y, Clayman GL. Sorafenib potently inhibits papillary thyroid carcinomas harboring RET/PTC1 rearrangement. Clin Cancer Res 2008;14:4908-14

66. Koh YW, Shah MH, Agarwal K, et al. Sorafenib and Mek inhibition is synergistic in medullary thyroid carcinoma in vitro. Endocr Relat Cancer 2012;19:29-38

67. Kim S, Yazici YD, Calzada G, et al. Sorafenib inhibits the angiogenesis and growth of 
orthotopic anaplastic thyroid carcinoma xenografts in nude mice. Mol Cancer Ther $2007 ; 6: 1785-92$

68. ** Gupta-Abramson V, Troxel AB, Nellore A, et al. Phase II trial of sorafenib in advanced thyroid cancer. J Clin Oncol 2008;26:4714-9. An important phase II trial of sorafenib in advanced thyroid cancer

69. ** Kloos RT, Ringel MD, Knopp MV, et al. Phase II trial of sorafenib in metastatic thyroid cancer. J Clin Oncol 2009;27:1675-84. An important phase II trial of sorafenib in advanced thyroid cancer

70. Hoftijzer H, Heemstra KA, Morreau H, et al. Beneficial effects of sorafenib on tumor progression, but not on radioiodine uptake, in patients with differentiated thyroid carcinoma. Eur J Endocrinol 2009;161:923-31

71. Brose MS, Troxel AB, Redlinger M, et al. Effect of BRAFV600E on response to sorafenib in advanced thyroid cancer patients. ASCO Annual Meeting, Orlando, Florida, 2009, J. Clin. Oncol. 2009

72. Cabanillas ME, Waguespack SG, Bronstein Y, et al. Treatment with tyrosine kinase inhibitors for patients with differentiated thyroid cancer: the M. D. Anderson Experience. J Clin Endocrinol Metab 2010;95:2588-95

73. Ahmed M, Barbachano Y, Riddell A, et al. Analysis of the efficacy and toxicity of sorafenib in thyroid cancer: a phase II study in a UK based population. Eur J Endocrinol $2011 ; 165: 315-22$

74. ** Keefe SM, Troxel AB, Rhee S, et al. Phase II trial of sorafenib in patients with advanced thyroid cancer. ASCO Annual Meeting, Pennsylvania, Philadelphia, 2011. J Clin Oncol 2011. An important phase II trial of sorafenib in advanced thyroid cancer

75. Capdevila J, Iglesias L, Halperin I, et al. Sorafenib in metastatic thyroid cancer. Endocr Related Cancer 2012;19:209-16

76. ** Schneider TC, Abdulrahman RM, Corssmit EP, et al. Long-term analysis of the efficacy 
and tolerability of sorafenib in advanced radio-iodine refractory differentiated thyroid carcinoma: final results of a phase II trial. Eur J Endocrinol 2012;167:643-50. An important phase II trial of sorafenib in advanced thyroid cancer

77. Savvides P, Nagaiah G, Lavertu PN, et al. Phase II Trial of Sorafenib in Patients with Advanced Anaplastic Carcinoma of the Thyroid. Thyroid 2013;23:600-4

78. * Marotta V, Ramando V, Camera L, et al. Sorafenib in advanced iodine-refractory differentiated thyroid cancer: efficacy, safety and exploratory analysis of role of serum thyroglobulin and FDG-PET. Clin Endocrinol (Oxf) 2013;78:760-7. Thyroglobulin and FDG-PET in patients with advanced thyroid carcinoma treated with sorafenib

79. Massicotte MH, Brassard M, Claude-Desroches M, et al. Tyrosine kinase inhibitor treatments in patients with metastatic thyroid carcinomas: a retrospective study of the TUTHYREF network. Eur J Endocrinol 2014;170:575-82

80. Benekli M, Yalcin S, Ozkan M, et al. Efficacy of sorafenib in advanced differentiated and medullary thyroid cancer: experience in a Turkish population. Onco Targets Ther 2014;8:15

81. Gallo M, Michelon F, Castiglione A, et al. Sorafenib treatment of radioiodine-refractory advanced thyroid cancer in daily clinical practice: a cohort study from a single center. Endocrine. 2014 Nov 21. [Epub ahead of print]

82. ** Brose MS, Nutting CM, Jarzab B, et al. Sorafenib in radioactive iodine-refractory, locally advanced or metastatic differentiated thyroid cancer: a randomised, double-blind, phase 3 trial. Lancet 2014;384:319-28. An important phase III trial of sorafenib in advanced thyroid cancer

83. Bayer. Nexavar ${ }^{\circledR}$ versus placebo in locally advanced/metastatic RAI-refractory differentiated thyroid cancer [ClinicalTrials.gov identifier: NCT00984282] (2015). https://clinicaltrials.gov/ct2/show/NCT00984282?term=NCT00984282\&rank=1

84. Gild ML, Bullock M, Robinson BG, Clifton-Bligh R. Multikinase inhibitors: a new option 
for the treatment of thyroid cancer. Nat Rev Endocrinol 2011;7:617-24

85. Grandinetti CA, Goldspiel BR. Sorafenib and sunitinib: novel targeted therapies for renal cell cancer. Pharmacotherapy $2007 ; 27: 1125-44$

86. Chan D, Zheng Y, Tyner JW, et al. Belinostat and panobinostat (HDACI): in vitro and in vivo studies in thyroid cancer. J Cancer Res Clin Oncol 2013;139:1507-14

87. Antonelli A, Ferrari SM, Fallahi P, et al. Thiazolidinediones and antiblastics in primary human anaplastic thyroid cancer cells. Clin Endocrinol (Oxf) 2009;70:946-53

88. Antonelli A, Bocci G, La Motta C, et al. CLM94, a novel cyclic amide with anti-VEGFR-2 and antiangiogenic properties, is active against primary anaplastic thyroid cancer in vitro and in vivo. J Clin Endocrinol Metab 2012;97:E528-E536

89. Newell DR. Flasks, fibres and flanks--pre-clinical tumour models for predicting clinical antitumour activity. Br J Cancer 2001;84:1289-90

90. Schroyens W, Tueni E, Dodion P, et al. Validation of clinical predictive value of in vitro colorimetric chemosensitivity assay in head and neck cancer. Eur J Cancer 1990;26:834-8

91. Antonelli A, Ferrari SM, Fallahi P, et al. Evaluation of the sensitivity to chemotherapeutics or thiazolidinediones of primary anaplastic thyroid cancer cells obtained by fine-needle aspiration. Eur J Endocrinol 2008;159:283-91

92. Antonelli A; Ferrari SM, Fallahi P, et al. Primary cell cultures from anaplastic thyroid cancer obtained by fine-needle aspiration used for chemosensitivity tests. Clin Endocrinol (Oxf) 2008;69:148-52

93. Antonelli A, Bocci G, Fallahi P, et al. CLM3, a multitarget tyrosine kinase inhibitor with antiangiogenic properties, is active against primary anaplastic thyroid cancer in vitro and in vivo. J Clin Endocrinol Metab 2014;99:E572-E81

94. Antonelli A, Bocci G, La Motta C, et al. Novel pyrazolopyrimidine derivatives as tyrosine kinase inhibitors with antitumoral activity in vitro and in vivo in papillary dedifferentiated thyroid cancer. J Clin Endocrinol Metab 2011;96:E288-E96 
95. Marotta V, Colao A, Faggiano A. Complete disappearance of liver metastases in a patient with iodine-refractory differentiated thyroid cancer subjected to sorafenib re-challenge. Endocrine 2015 Mar 10. [Epub ahead of print] 
Table I. Phase II trials of sorafenib in patients with thyroid cancer.

\begin{tabular}{|c|c|c|c|c|c|c|}
\hline \multirow[t]{2}{*}{ Drug } & \multirow{2}{*}{$\begin{array}{l}\text { Type of } \\
\text { Thyroid } \\
\text { cancer }\end{array}$} & \multicolumn{4}{|c|}{ Responses } & \multirow[t]{2}{*}{ Authors } \\
\hline & & $P R$ & $S D$ & $P D$ & $\begin{array}{c}P F S \\
\text { (months) }\end{array}$ & \\
\hline Sorafenib & $30 \mathrm{DeTC}$ & $23.3 \%$ & $53 \%$ & $7 \%$ & 21 & $\begin{array}{c}\text { Gupta-Abramson et } \\
\text { al. [68] }\end{array}$ \\
\hline Sorafenib & $41 \mathrm{DeTC}$ & $15 \%$ & $56 \%$ & - & 15 & Kloos et al. [69] \\
\hline Sorafenib & 31 DeTC & $25 \%$ & $34 \%$ & $22 \%$ & 14.5 & Hoftijzer et al. [70] \\
\hline Sorafenib & 13 DeTC & $20 \%$ & $60 \%$ & $20 \%$ & 19 & Cabanillas et al. [72] \\
\hline Sorafenib & $\begin{array}{l}19 \text { DeTC } \\
15 \text { MTC }\end{array}$ & $21 \%$ & $65 \%$ & $14 \%$ & & Ahmed et al. [73] \\
\hline Sorafenib & $\begin{array}{c}47 \text { DeTC } \\
5 \text { ATC } \\
3 \text { MTC }\end{array}$ & $38 \%$ DeTC & 47\% DeTC & - & 23.4 & Keefe et al. [74] \\
\hline Sorafenib & $\begin{array}{c}16 \text { DeTC } \\
15 \text { MTC } \\
3 \text { ATC }\end{array}$ & $32 \%$ & $41 \%$ & $\begin{array}{r}25 \% \\
7 \% \\
66 \%\end{array}$ & $\begin{array}{c}13.5 \mathrm{DeTC} \\
10.5 \mathrm{MTC} \\
4.4 \mathrm{ATC}\end{array}$ & Capdevila et al. [75] \\
\hline Sorafenib & 31 DeTC & $31 \%$ & $42 \%$ & $58 \%$ & 18 & Schneider et al. [76] \\
\hline Sorafenib & $20 \mathrm{ATC}$ & $10 \%$ & $25 \%$ & - & 1.9 & Savvides et al. [77] \\
\hline Sorafenib & 17 DeTC & $30 \%$ & $41 \%$ & $18 \%$ & 9 & Marotta et al. [78] \\
\hline $\begin{array}{c}\text { Sorafenib } \\
\text { Sunitinib } \\
\text { Vandetani } \\
\text { b }\end{array}$ & $\begin{array}{l}32 \text { DeTC } \\
13 \text { ATC } \\
17 \text { MTC }\end{array}$ & $\begin{array}{c}15 \% \text { vs } 8 \% \\
\text { DeTC } \\
36 \% \text { MTC }\end{array}$ & - & - & $\begin{array}{l}6.7 \text { vs } 7 \\
\text { DeTC }\end{array}$ & Massicotte et al. [79] \\
\hline
\end{tabular}




\begin{tabular}{|c|c|c|c|c|c|c|}
\hline Sorafenib & 14 DeTC & $20 \%$ & $33 \%$ & - & 21.3 DeTC & Benekli et al. [80] \\
& 16 MTC & & & & 14.5 MTC & \\
\hline
\end{tabular}

DeTC: Dedifferentiated Thyroid Cancer; MTC: Medullary Thyroid Cancer; ATC: Anaplastic Thyroid Cancer PR: Partial Response; SD: Stable Disease; PD: Progressive Disease; PFS: Progression Free Survival 
Table II. Sorafenib undesirable effects.

\begin{tabular}{|c|c|c|c|c|}
\hline \multirow{2}{*}{$\begin{array}{c}\text { Classification } \\
\text { (system or organ) }\end{array}$} & \multicolumn{4}{|c|}{ Undesirable effects incidence } \\
\hline & $\begin{array}{l}\text { Very common } \\
(\geq 1 / 10)\end{array}$ & $\begin{array}{c}\text { Common } \\
\geq 1 / 100,<1 / 10) \\
\end{array}$ & $\begin{array}{c}\text { Uncommon } \\
(\geq 1 / 1000,<1 / 100)\end{array}$ & $\begin{array}{c}\text { Rare } \\
(\geq 1 / 10000 \text { to }<1 / 1000)\end{array}$ \\
\hline $\begin{array}{l}\text { Hemolymphopoietic } \\
\text { Diseases }\end{array}$ & Lymphopenia & $\begin{array}{c}\text { Leukopenia } \\
\text { Neutropenia } \\
\text { Thrombocytopenia } \\
\end{array}$ & & \\
\hline Vascular diseases & $\begin{array}{l}\text { Hypertension, bleeding } \\
\text { (from the } \\
\text { gastrointestinal tract, } \\
\text { respiratory tract; } \\
\text { cerebral haemorrhage) }\end{array}$ & & Hypertensive crisis & \\
\hline Cardiac diseases & & $\begin{array}{c}\text { Congestive heart } \\
\text { failure, myocardial } \\
\text { ischaemia }\end{array}$ & & QT prolungation \\
\hline $\begin{array}{l}\text { Respiratory tract and } \\
\text { mediastinic diseases }\end{array}$ & & Raucousness & $\begin{array}{c}\text { Rhinorrhea, interstitial } \\
\text { pneumonial like } \\
\text { diseases }\end{array}$ & \\
\hline Renal diseases & & Renal failure & & \\
\hline $\begin{array}{l}\text { Gastrointestinal tract } \\
\text { diseases }\end{array}$ & $\begin{array}{c}\text { Diarrhea, nausea, } \\
\text { vomit }\end{array}$ & & $\begin{array}{l}\text { Gastro-esophageal } \\
\text { reflux, gastritis, } \\
\text { pancreatitis, bowel } \\
\text { perforation } \\
\end{array}$ & \\
\hline $\begin{array}{l}\text { Hepatobiliary } \\
\text { diseases }\end{array}$ & & & Jaundice, cholecystitis & Drug related \\
\hline $\begin{array}{l}\text { Metabolic and } \\
\text { nutritional disorders }\end{array}$ & Hypophosphatemia & $\begin{array}{c}\text { Anorexia } \\
\text { Hypocalcemia }\end{array}$ & $\begin{array}{l}\text { Hyponatriemia } \\
\text { Dehydration }\end{array}$ & \\
\hline Endocrine disorders & & & Hypo/hyperthyroidism & \\
\hline $\begin{array}{l}\text { Diseases of } \\
\text { reproductive system } \\
\text { and breast }\end{array}$ & & Erectile dysfunction & Gynecomastia & \\
\hline $\begin{array}{l}\text { Neurological } \\
\text { Diseases }\end{array}$ & & $\begin{array}{c}\text { Periferical sensorial } \\
\text { neurophaty }\end{array}$ & $\begin{array}{c}\text { Reversible white } \\
\text { matter encephalopathy }\end{array}$ & \\
\hline Ear disease & & Tinnitus & & \\
\hline $\begin{array}{l}\text { Muscle skeletal } \\
\text { system and } \\
\text { connettive tissue } \\
\text { disease }\end{array}$ & & Arthralgia, myalgia & & Rhabdomyolysis \\
\hline $\begin{array}{l}\text { Immune system } \\
\text { disorders }\end{array}$ & & & $\begin{array}{l}\text { Hypersensivity (skin } \\
\text { reaction and nettle } \\
\text { rash) }\end{array}$ & $\begin{array}{c}\text { Angioedema, } \\
\text { anaphylaxis shock }\end{array}$ \\
\hline Skin & $\begin{array}{l}\text { Rash, alopecia, hand- } \\
\text { foot syndrome, itch }\end{array}$ & $\begin{array}{c}\text { Skin dryness, } \\
\text { exfoliative dermatitis, } \\
\text { acne } \\
\end{array}$ & $\begin{array}{l}\text { Erythema multiforme, } \\
\text { eczema }\end{array}$ & $\begin{array}{c}\text { Stevens Johnsons } \\
\text { syndrome, vasculitis }\end{array}$ \\
\hline Infections & & & $\begin{array}{l}\text { Infection } \\
\text { Folliculitis }\end{array}$ & \\
\hline Psychiatric disorder & & Depression & & \\
\hline Other diseases & $\begin{array}{l}\text { Fatigue, pain (mouth, } \\
\text { abdomen, bone, } \\
\text { oncologic), headache }\end{array}$ & $\begin{array}{l}\text { Asthenia, fever, } \\
\text { influenza like } \\
\text { syndrome }\end{array}$ & & \\
\hline Laboratory exams & $\begin{array}{l}\text { Increasing levels of } \\
\text { amylase and lipase }\end{array}$ & $\begin{array}{c}\text { Weight loss, increasing } \\
\text { levels of hepatic } \\
\text { enzyme }\end{array}$ & $\begin{array}{c}\text { Alteration of INR } \\
\text { value }\end{array}$ & \\
\hline
\end{tabular}

$I N R=$ International Normalized Ratio 